\title{
Advances and Future Directions in Molecular Breast Imaging
}

\author{
Matthew F. Covington ${ }^{1}$, Ephraim E. Parent ${ }^{2}$, Elizabeth H. Dibble ${ }^{3}$, Gaiane M. Rauch ${ }^{4}$, and Amy M. Fowler ${ }^{5}$ \\ ${ }^{I}$ Center for Quantitative Cancer Imaging, Huntsman Cancer Institute and University of Utah Department of Radiology and Imaging \\ Sciences, Salt Lake City, Utah, ${ }^{2}$ Mayo Clinic Department of Radiology, Jacksonville, Florida $;{ }^{3}$ Warren Alpert Medical School of Brown \\ University/Rhode Island Hospital Department of Diagnostic Imaging, Providence, Rhode Island; ${ }^{4}$ M.D. Anderson Cancer Center, \\ Departments of Abdominal and Breast Imaging, Houston, Texas; and ${ }^{5}$ University of Wisconsin School of Medicine and Public Health, \\ Departments of Radiology and Medical Physics and the University of Wisconsin Carbone Cancer Center, Madison, Wisconsin
}

\begin{abstract}
Molecular breast imaging (MBI) using ${ }^{99 m}$ Tc-sestamibi has advanced rapidly over the past decade. Technical advances allow lower-dose, higher-resolution imaging and biopsy capability. MBI can be used for supplemental breast cancer screening with mammography for women with dense breasts, as well as to assess neoadjuvant therapy response, evaluate disease extent, and predict breast cancer risk. This article highlights the current state of the art and future directions in MBI.
\end{abstract}

Key Words: molecular breast imaging; MBI; breast specific $\gamma$-imaging; nuclear breast imaging; sestamibi

J Nucl Med 2022; 63:17-21

DOI: 10.2967/jnumed.121.261988

$\mathbf{T}$ he term molecular breast imaging (MBI) may broadly refer to all nuclear medicine and advanced MRI techniques for breast imaging. However, MBI usually refers to dedicated breast imaging systems using ${ }^{99 \mathrm{~m}} \mathrm{Tc}$-sestamibi with dual-head solid-state cadmiumzinc-telluride (CZT) detectors. Compared with breast-specific $\gamma$-imaging that uses single-head scintillation detectors, MBI uses dual-head CZT detectors and advanced collimator designs to improve spatial resolution, shorten imaging times, and facilitate lower-dose imaging. This article summarizes the current state of the art and the future uses of MBI.

\section{CURRENT STATE OF THE ART}

There are 2 commercially available MBI systems: LumaGem (CMR Naviscan) and Eve Clear Scan (SmartBreast Corp.). Both use CZT semiconductor detectors that directly convert $\gamma$-photons into electrons. Compared with single-head breast-specific $\gamma$-imaging systems, dual-head CZT detectors improve detection of small lesions (1).

The primary radiopharmaceutical used for MBI is ${ }^{99 \mathrm{~m}} \mathrm{Tc}$-sestamibi. ${ }^{99 \mathrm{~m}} \mathrm{Tc}$-sestamibi uptake in breast tumors results from increased mitochondrial density, increased blood flow, and negative membrane potentials of tumor cells (2). ${ }^{99 \mathrm{~m}} \mathrm{Tc}$-sestamibi has been used in hundreds of thousands of patients with a favorable safety profile and few contraindications, namely pregnancy and a

\footnotetext{
Received Jun. 1, 2021; revision accepted Nov. 16, 2021.

For correspondence or reprints, contact Matthew F. Covington (matthew. covington@hsc.utah.edu).

COPYRIGHT (C) 2022 by the Society of Nuclear Medicine and Molecular Imaging.
}

prior allergic reaction to ${ }^{99 \mathrm{~m}} \mathrm{Tc}$-sestamibi (2). Patients who are breast feeding may continue to do so, unless otherwise specified by institutional guidelines. ${ }^{99 \mathrm{~m}} \mathrm{Tc}$-sestamibi clears from the bloodstream rapidly within $2-3 \mathrm{~min}$; thus, image acquisition can begin within 5 min after injection (3). Having the patients fast, warming them with a blanket, and having them keep still may reduce background breast uptake (4).

Standard craniocaudal and mediolateral oblique projections of each breast are acquired using gentle compression, with 7-10 min per view (4). Breast positioning is comparable to mammography, aiding correlation of findings between mammography and MBI. MBI detector size is $20 \times 16 \mathrm{~cm}$ or $24 \times 16 \mathrm{~cm}$, compared with $24 \times 29 \mathrm{~cm}$ for mammography. Tiled acquisitions may be required with MBI if the breast size exceeds the field of view. Lesions near the chest wall and axillary lymph nodes may be difficult to visualize with MBI, because of increased dead space at the edge of the field of view (8 mm) compared with mammography (4 mm) (4). Furthermore, MBI detectors are opaque, making maximal posterior positioning difficult to confirm (4). The patient can breathe normally during imaging, with instructions to otherwise remain still to reduce motion blur. Performing screening MBI during the follicular phase may minimize background parenchymal uptake (BPU), and menstrual cycle phase may be included in the report (5). However, no studies have examined menstrual cycle timing and MBI diagnostic performance, and recent data from screening breast MRI outcomes indicate that scheduling based on menstrual cycle phase is not necessary (6). MBI should not be scheduled within 4 half-lives of other ${ }^{99 \mathrm{~m}} \mathrm{Tc}$-based studies, PET/CT, or targeted radionuclide therapies because of photon energy window overlap.

\section{RADIATION EXPOSURE}

The absorbed radiation dose during MBI is proportional to the administered activity of ${ }^{99 \mathrm{~m}} \mathrm{Tc}$-sestamibi. Optimizations in detector design, patient preparation, and tracer delivery have reduced the administered activity from $740-1,100 \mathrm{MBq}(20-30 \mathrm{mCi})$ to the current off-label standard of $240-300 \mathrm{MBq}(6.5-8 \mathrm{mCi})(7,8)$. Weight-based adjustments for injected activity are not necessary for MBI (9). The average absorbed radiation dose to the breast from $300 \mathrm{MBq}(8 \mathrm{mCi})$ of ${ }^{99 \mathrm{~m}} \mathrm{Tc}$-sestamibi is estimated to be $1.1 \mathrm{mGy}$, compared with $3.0-4.5 \mathrm{mGy}$ with mammography and tomosynthesis.

Since ${ }^{99 \mathrm{~m}} \mathrm{Tc}$-sestamibi is systemically distributed, tissues outside the breast receive the largest radiation dose. The estimated effective (whole-body) dose for $300 \mathrm{MBq}(8 \mathrm{mCi})$ of ${ }^{99 \mathrm{~m}} \mathrm{Tc}$-sestamibi is $2.1-2.6 \mathrm{mSv}$, which is at, or lower than, annual natural 
background levels ( $\sim 3 \mathrm{mSv})(10)$. For reference, the effective dose of chest CT can approach $6 \mathrm{mSv}$ (11). Tissues with the highest exposures include the colon ( $7.1 \mathrm{mGy})$, urinary bladder $(3.2 \mathrm{mGy})$, and gallbladder (11.5 mGy) (12). Various organizations assessing radiation risk and radiation protection (Health Physics Society, American Association of Physicists in Medicine, International Organization for Medical Physics, and United Nations Scientific Committee on the Effects of Atomic Radiation) state that risks from radiation doses of less than $100 \mathrm{mSv}$ are not significantly different from zero (10). Thus, current MBI radiation exposure is deemed to pose negligible risk to the patient, with minimal theoretic risk of inducing cancer in any of these organs (10).

The administered activity of ${ }^{99 \mathrm{~m}} \mathrm{Tc}$-sestamibi for MBI continues to decrease with technologic advancement. Tao et al. showed that new image-processing algorithms maintain lesion conspicuity with a simulated half-dose (150 MBq [4 mCi]) injection (13). Continued advances in CZT module design will improve sensitivity and should allow further dose reduction.

\section{INDICATIONS AND CLINICAL APPLICATIONS}

\section{Supplemental Screening}

Approximately $43 \%$ of the 40 million individuals in the U.S. screening population have dense breast tissue (14-16). Screening mammography has relatively reduced sensitivity for breast cancer detection in individuals with dense breasts (15). Furthermore, breast cancer risk is higher for individuals with dense breasts $(15,17)$. Supplemental screening may therefore benefit 16-20 million U.S. individuals with dense breasts who are at risk for false-negative results on screening mammography. Breast cancer detection with supplemental screening methods such as whole-breast ultrasound, breast MRI, contrast-enhanced mammography, and MBI is not limited by dense breast tissue (15).

The ideal supplemental screening modality would have a high breast cancer detection rate and a high negative predictive value while maintaining safety, affordability, ease of access, quick imaging acquisition and interpretation times, and acceptable rates of false-positive findings. Although MBI addresses some of these issues, it also has some limitations as a screening test. MBI excels with high lesion-to-background contrast in individuals with dense breasts or breast implants (18), with an incremental cancer detection rate of 8.8 per 1,000 exams on the first MBI screening (8). For comparison, the baseline detection rate of screening mammography is approximately 3.2 cancers per 1,000 individuals screened (19). The incremental cancer detection rate per 1,000 exams in individuals with dense breasts is approximately 1.7 for tomosynthesis, 2.7 for ultrasound, 15 for full-protocol MRI, and 10 for abbreviated MRI (15). MBI also provides favorable false-positive rates (20), favorable recall rates (8), rapid interpretation times (18), low cost (15), few contraindications (21), and a high negative predictive value (22). Disadvantages of MBI for supplemental screening include an imaging exam time of 28-40 min (15), the need for nuclear medicine licensing and coordination between nuclear medicine and breast imaging sections, lack of widespread availability, variable insurance coverage, and a higher whole-body radiation exposure than for other supplemental screening options $(10,13,15)$.

When used for supplemental screening, the estimated benefit of MBI in terms of deaths averted is 5-9 times greater than the estimated risk of lives lost (23), and the estimated benefit-to-radiation risk ratio now approaches that of mammography (23). However, concerns regarding MBI radiation risks, though disputed, have delayed widespread use for supplemental screening $(10,21,23)$. The American College of Radiology appropriateness criteria for supplemental breast cancer screening based on breast density currently do not recommend MBI (24).

The prospective multicenter Density MATTERS trial compares MBI with tomosynthesis for supplemental screening in women with dense breasts. This is the first trial to examine incidence screening MBI in which performance is evaluated not only for the initial scan but also at a subsequent screening round. Preliminary results show an incremental cancer detection rate for MBI beyond tomosynthesis of 9.3 per 1,000 screened, with 6 invasive cancers seen only on MBI (median size, $1 \mathrm{~cm}$; 5 of 6 lymph node-negative) among 537 of the planned 3,000 participants (25). By comparison, the Eastern Cooperative Oncology Group/American College of Radiology Imaging Network EA1141 trial of abbreviated breast MRI for screening women with dense breasts found that abbreviated breast MRI after tomosynthesis had an incremental invasive cancer detection rate of of 7 per 1,000 screened (26). Thus, MBI may detect invasive breast cancers occult on tomosynthesis in women with dense breasts at a rate similar to that of abbreviated breast MRI. Studies of women invited to undergo screening breast MRI showed that $41 \%-42 \%$ declined participation for reasons including lower socioeconomic status, self-reported contraindications to MRI (27), claustrophobia, and financial concerns (28). MBI may be considered an alternative for such individuals.

\section{Local Tumor Extent}

For newly diagnosed breast cancer, accurate determination of disease extent is important for defining clinical stage and for planning therapy and surgery. MBI can be used for local tumor staging to detect multifocal, multicentric, or contralateral malignancy, especially for patients who cannot obtain preoperative breast MRI (Fig. 1). A study comparing extent of disease using MBI, contrastenhanced mammography, and MRI found that MBI and contrastenhanced mammography were effective for local staging, with similar visualization of the index cancers and increased specificity for additional cancers compared with MRI (20). However, unlike MRI, MBI is limited for evaluation of axillary and chest wall disease. In a study of 90 patients with breast cancer before starting neoadjuvant chemotherapy (NAT), MBI detected 16 of 20 tumors smaller than $1 \mathrm{~cm}$, compared with 17 of 20 tumors for MRI (29). An additional limitation of MBI for surgical planning regards invasive lobular carcinomas, which have less intense sestamibi uptake than invasive ductal carcinomas, resulting in lower detection (30).

\section{NAT}

NAT is often the standard of care for locally advanced breast cancer. NAT can reduce tumor burden, allowing less extensive surgery. MBI is relatively accurate for prediction of pathologic response and evaluation of residual disease after NAT (Fig. 2). A metaanalysis of 14 studies with 529 breast cancer patients found that MBI had a sensitivity of $70.3 \%(95 \% \mathrm{CI}, 56.5 \%-81.3 \%)$ and a specificity of $90.1 \%(95 \% \mathrm{CI}, 77.5 \%-96.0 \%)$ for residual disease (31). A retrospective report of 114 patients who underwent NAT found slightly lower sensitivity for MBI than for MRI $(70 \%$ vs. $83 \%$ ) but higher specificity ( $90 \%$ vs. $60 \%$ ) for residual disease, with similar overall performance (respective $\kappa$-values, 0.47 vs. $0.41[P<0.001])(32)$. A prospective study of 104 patients undergoing NAT found that MBI had lower sensitivity than MRI 


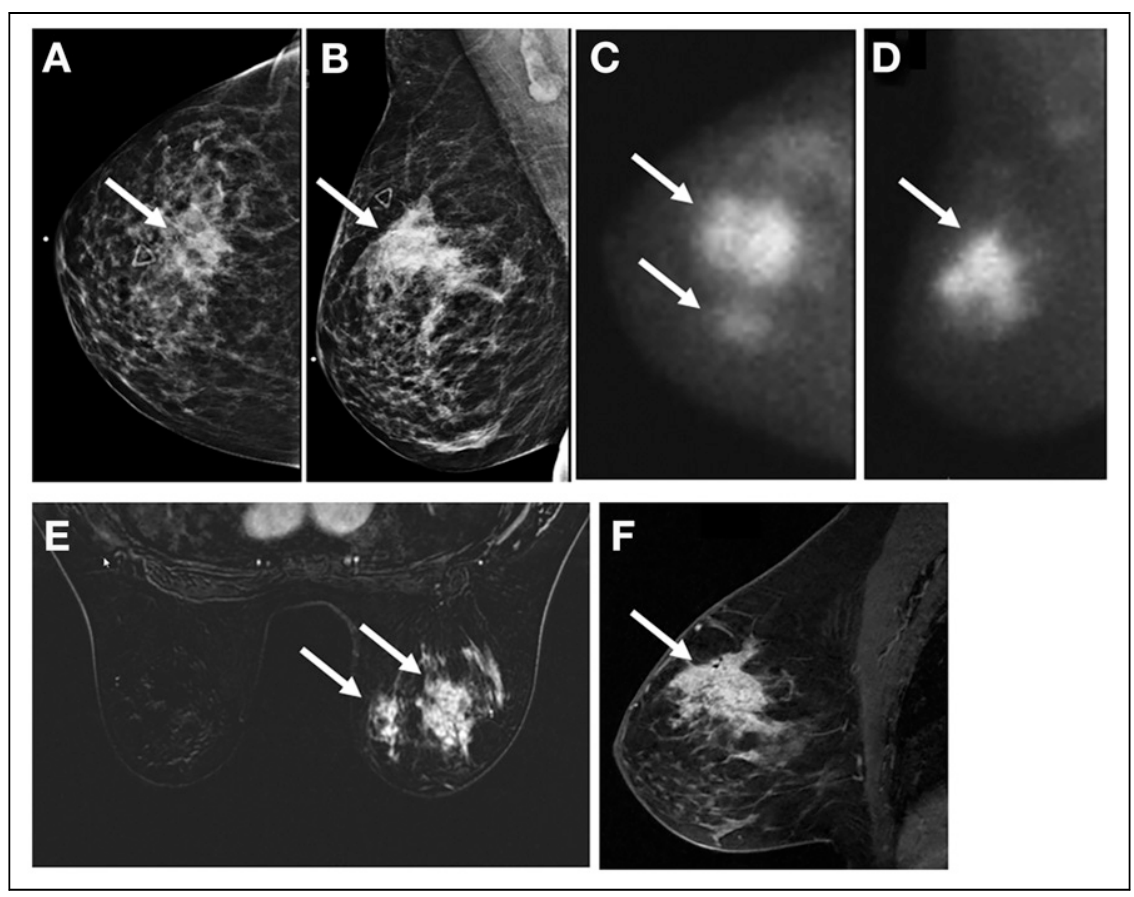

FIGURE 1. MBI with $300 \mathrm{MBq}(8 \mathrm{mCi})$ of ${ }^{99 \mathrm{~m}} \mathrm{Tc}$-sestamibi for extent-of-disease evaluation in $59-\mathrm{y}$ old woman with palpable irregular mass (arrows) in right upper central breast. ( $A$ and $B$ ) Mass measured $3.3 \mathrm{~cm}$ on craniocaudal $(A)$ and mediolateral oblique (B) mammograms. (C and D) MBI showed $10 \mathrm{~cm}$ of uptake on craniocaudal (C) and mediolateral oblique (D) views. (E and F) Postcontrast axial (E) and sagittal (F) MRI confirmed $10.2 \mathrm{~cm}$ of abnormal enhancement. After NAT and mastectomy, surgical pathology showed $8-\mathrm{cm}$ treated tumor bed with $0.2 \mathrm{~cm}$ of residual invasive carcinoma.

\section{PRESENT AND FUTURE DEVELOPMENTS}

\section{MBI-Guided Biopsy}

For suggestive MBI findings, biopsy is necessary to confirm malignancy. For MBIdetected masses, targeted ultrasound and ultrasound-guided biopsy are performed, paralleling MRI workflows. In a study of 1,585 examinations performed before the availability of MBI biopsy, 115 MBIpositive findings were detected, resulting in 50 biopsy recommendations. Of these 50 lesions recommended for biopsy on the basis of MBI, $38(76 \%)$ were visible sonographically, but $12(24 \%)$ required MBI biopsy (34). The billed expense of MRIguided biopsy is estimated at $\$ 3,500$, whereas MBI-guided biopsy is roughly half this amount (35).

MBI-directed biopsy is currently available for 1 system (Eve Clear Scan). This unit is a self-contained accessory mounted on the dual-head system and includes an angled pair of CZT detectors for obtaining stereotactic views, allowing specimen imaging to confirm adequate sampling. A breastspecific $\gamma$-imaging-guided biopsy system has also been described (36). MRI-guided biopsy can be used if an MRI correlate is identified, but it does not allow specimen imaging.
(58.9\% vs. $82.8 \%$ ) but improved specificity ( $82.4 \%$ vs. $69.4 \%)$ in evaluating residual disease (29). The accuracy of MBI for residual disease assessment depends on tumor molecular subtype, with the highest accuracy for triple-negative and human epidermal growth factor receptor 2-positive subtypes and the lowest accuracy for luminal subtypes (33). However, no imaging technique is currently able to definitively determine complete pathologic response to therapy without surgical confirmation, in part because of inherent resolution limitations.

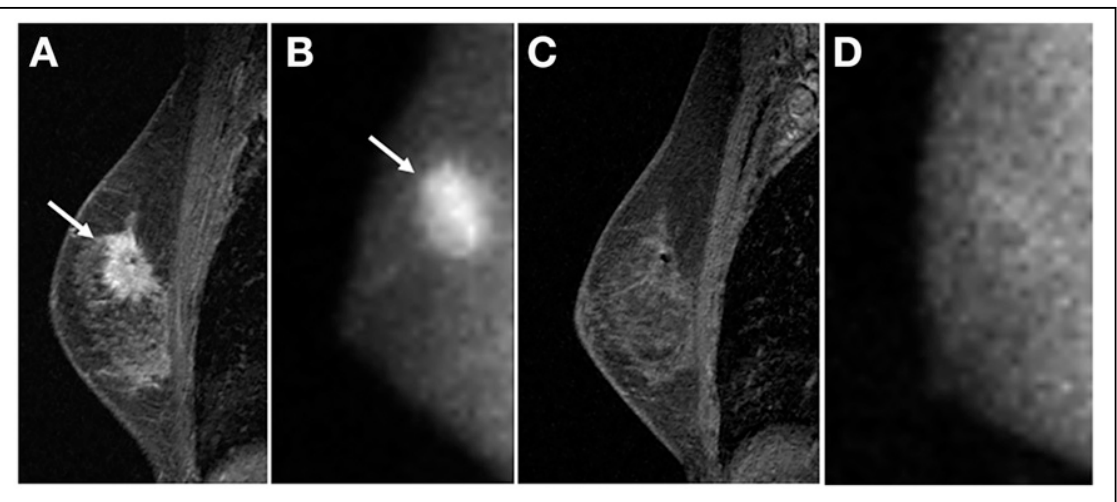

FIGURE 2. A 38-y-old woman with right-breast triple-negative and node-negative invasive ductal carcinoma (arrows). (A and B) Pretreatment postcontrast sagittal fat-suppressed T1-weighted MRI shows irregular mass in right breast (A), and MBI with $300 \mathrm{MBq}(8 \mathrm{mCi})$ of ${ }^{99 \mathrm{~m}} \mathrm{Tc}$-sestamibi shows intense uptake in mediolateral oblique view (B). (C and $D)$ On posttreatment imaging, there is no residual enhancement on MRI (C) and no residual uptake on MBI (D). Surgical pathology showed pathologic complete response.

\section{Breast Cancer Risk Assessment}

Numerous models exist for predicting an individual's breast cancer risk (37). These models inform whether and when individuals should undergo high-risk screening and potentially benefit from risk-reducing endocrine therapy or prophylactic mastectomy.

Imaging biomarkers are increasingly used for risk stratification (38). Breast density is an anatomic imaging biomarker associated with increased breast cancer risk $(15,39)$. Growing evidence suggests that functional imaging modalities, such as MBI and MRI, can predict breast cancer risk (40-42), perhaps more accurately than risk models alone (42). BPU on MBI is a reliable quantitative and qualitative biomarker $(41,43)$ that describes the amount of radiotracer uptake in normal breast tissue relative to subcutaneous fat. BPU is assessed qualitatively as photopenic, minimal to mild, moderate, or marked (Fig. 3) (44). BPU is analogous to background parenchymal enhancement on MRI, which describes the amount of enhancement in normal breast tissue and is similarly assessed qualitatively as minimal, mild, moderate, or marked (45). Beyond predicting breast cancer risk, BPU varies with menopausal status and dose of riskreduction endocrine therapy (46). A large single-center study found that BPU is an independent risk factor for breast cancer in postmenopausal women with elevated BPU 


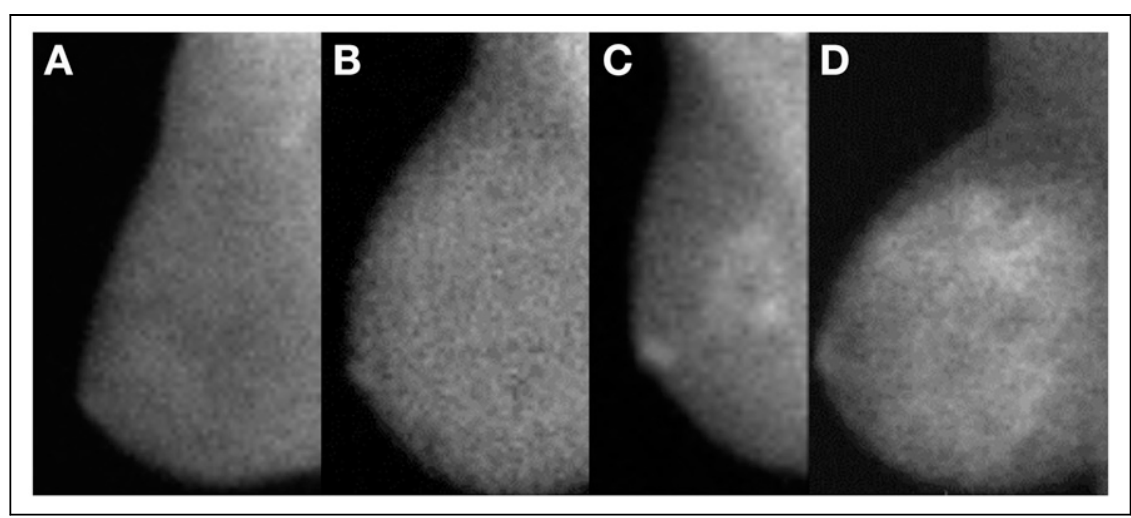

FIGURE 3. Different degrees of $\mathrm{BPU}$ on $\mathrm{MBI}\left(300 \mathrm{MBq}\right.$ [8 mCi] of ${ }^{99 \mathrm{~m}} \mathrm{Tc}-$-sestamibi): photopenic (fibroglandular uptake less intense than fat uptake) (A), minimal to mild (fibroglandular uptake equal to, or just noticeably more intense than, fat uptake) (B), moderate (fibroglandular uptake more than mild but less than twice as intense as fat uptake) (C), and marked (fibroglandular uptake at least twice as intense as fat uptake) (D).

and dense breasts at highest risk, well above the threshold for considering use of risk-reducing therapy (47). Future studies incorporating functional imaging biomarkers into breast cancer risk models may facilitate personalized screening and risk management.

\section{Novel Radiotracers}

MBI systems can also be used for research using $\gamma$-emitting radionuclides targeting more specific aspects of tumor biology beyond ${ }^{99 \mathrm{~m}}$ Tc-sestamibi uptake. Radiolabeled antibodies, peptides, and receptor ligands may broaden future applications of MBI for tumor phenotyping, neoadjuvant treatment selection, and therapy response prediction. Use of MBI for $\alpha_{V} \beta_{3}$ integrin-targeted imaging of tumor angiogenesis has been explored in 2 small studies $(48,49)$. Other ${ }^{99 \mathrm{~m}} \mathrm{Tc}-$ labeled radiotracers targeting specific receptors such as estrogen receptor and human epidermal growth factor receptor 2 have been developed and could use MBI technology but have not been evaluated yet.

\section{CONCLUSION}

MBI is an important emerging breast imaging technology for supplemental screening, local tumor staging, neoadjuvant therapy response assessment, and breast cancer risk assessment.

\section{DISCLOSURE}

Matthew Covington is a consultant for Invicro, LLC, for medical imaging review. Ephraim Parent receives research funding from Blue Earth Diagnostics. Gaiane Rauch receives research funding from GE Healthcare. Amy Fowler receives research support from GE Healthcare and book chapter royalties from Elsevier, Inc. All disclosures are outside the scope of this project. No other potential conflict of interest relevant to this article was reported.

\section{REFERENCES}

1. Hruska CB, Phillips SW, Whaley DH, Rhodes DJ, O'Connor MK. Molecular breast imaging: use of a dual-head dedicated gamma camera to detect small breast tumors. AJR. 2008;191:1805-1815.

2. Maublant J. Uptake Mechanisms of ${ }^{99 m}$ Tc-Labeled Perfusion Imaging Agents in Detection of Breast Cancers. CRC Press; 1998:327-338.

3. Hruska CB. Molecular breast imaging for screening in dense breasts: state of the art and future directions. AJR. 2017;208:275-283.
4. Swanson TN, Tran TD, Ellingson LR, et al. Best practices in molecular breast imaging: a guide for technologists. J Nucl Med Technol. 2018;46: 3-11.

5. Hruska CB, Conners AL, Vachon CM, et al. Effect of menstrual cycle phase on background parenchymal uptake at molecular breast imaging. Acad Radiol. 2015;22:1147-1156.

6. Lee $\mathrm{CH}$, Bryce Y, Zheng J, et al. Outcome of screening MRI in premenopausal women as a function of the week of the menstrual cycle. AJR. 2020; 214:1175-1181.

7. Shermis RB, Wilson KD, Doyle MT, et al. Supplemental breast cancer screening with molecular breast imaging for women with dense breast tissue. AJR. 2016;207:450-457.

8. Dibble EH, Hunt KN, Ehman EC, O'Connor MK. Molecular breast imaging in clinical practice. AJR. 2020;215:277-284.

9. Tran TD, Ellingson LR, Swanson TN, Solberg CM, O'Connor MK, Hruska CB. Molecular breast imaging: administered activity does not require adjustment based on patient size. J Nucl Med Technol. 2018;46:265-267.

10. Hruska CB. Let's get real about molecular breast imaging and radiation risk. Radiol Imaging Cancer. 2019;1:e190070.

11. Mettler FA Jr, Huda W, Yoshizumi TT, Mahesh M. Effective doses in radiology and diagnostic nuclear medicine: a catalog. Radiology. 2008;248:254-263.

12. Mattsson S, Johansson L, Leide Svegborn S, et al. Radiation dose to patients from radiopharmaceuticals: a compendium of current information related to frequently used substances. Ann ICRP. 2015;44:7-321.

13. Tao AT, Hruska CB, Conners AL, et al. Dose reduction in molecular breast imaging with a new image-processing algorithm. AJR. 2020;214:185-193.

14. 2019 Scorecard Statistics. U.S. Food and Drug Administration website. https:// www.fda.gov/radiation-emitting-products/mqsa-insights/2019-scorecard-statistics. Published 2019. Updated January 2, 2021. Accessed December 6, 2021.

15. Berg WA, Rafferty EA, Friedewald SM, Hruska CB, Rahbar H. Screening algorithms in dense breasts: AJR expert panel narrative review. AJR. 2021;216:275-294.

16. Sprague BL, Gangnon RE, Burt V, et al. Prevalence of mammographically dense breasts in the United States. J Natl Cancer Inst. 2014;106:dju255.

17. Buist DS, Porter PL, Lehman C, Taplin SH, White E. Factors contributing to mammography failure in women aged 40-49 years. J Natl Cancer Inst. 2004; 96:1432-1440.

18. Shermis RB, Redfern RE, Burns J, Kudrolli H. Molecular breast imaging in breast cancer screening and problem solving. Radiographics. 2017;37:1309-1606.

19. Rhodes DJ, Hruska CB, Conners AL, et al. Journal club: molecular breast imaging at reduced radiation dose for supplemental screening in mammographically dense breasts. AJR. 2015;204:241-251.

20. Sumkin JH, Berg WA, Carter GJ, et al. Diagnostic Performance of MRI, molecular breast imaging, and contrast-enhanced mammography in women with newly diagnosed breast cancer. Radiology. 2019;293:531-540.

21. Muzahir S. Molecular breast cancer imaging in the era of precision medicine. AJR. 2020;215:1512-1519.

22. Jain R, Katz DR, Kapoor AD. The clinical utility of a negative result at molecular breast imaging: initial proof of concept. Radiol Imaging Cancer. 2020;2:e190096.

23. Brown M, Covington MF. Comparative benefit-to-radiation risk ratio of molecular breast imaging, two-dimensional full-field digital mammography with and without tomosynthesis, and synthetic mammography with tomosynthesis. Radiol Imaging Cancer. 2019;1:e190005.

24. Weinstein SP, Slanetz PJ, Lewin AA, et al. ACR Appropriateness Criteria® supplemental breast cancer screening based on breast density. American College of Radiology website. https://acsearch.acr.org/docs/3158166/Narrative/. Accessed December 6, 2021.

25. Rhodes D, Hunt K, Conners A, et al. Molecular breast imaging and tomosynthesis to eliminate the reservoir of undetected cancer in dense breasts: the Density MATTERS trial [abstract]. Cancer Res. 2019;79:PD4-05.

26. Comstock CE, Gatsonis C, Newstead GM, et al. Comparison of abbreviated breast MRI vs digital breast tomosynthesis for breast cancer detection among women with dense breasts undergoing screening. JAMA. 2020;323:746-756.

27. de Lange SV, Bakker MF, Monninkhof EM, et al. Reasons for (non)participation in supplemental population-based MRI breast screening for women with extremely dense breasts. Clin Radiol. 2018;73:759.e1-759.e9.

28. Berg WA, Blume JD, Adams AM, et al. Reasons women at elevated risk of breast cancer refuse breast MR imaging screening: ACRIN 6666. Radiology. 2010;254: 79-87. 
29. Hunt KN, Conners AL, Goetz MP, et al. Comparison of ${ }^{99 \mathrm{~m}} \mathrm{Tc}$-sestamibi molecular breast imaging and breast MRI in patients with invasive breast cancer receiving neoadjuvant chemotherapy. AJR. 2019;213:932-943.

30. Conners AL, Jones KN, Hruska CB, Geske JR, Boughey JC, Rhodes DJ. Directconversion molecular breast imaging of invasive breast cancer: imaging features, extent of invasive disease, and comparison between invasive ductal and lobular histology. AJR. 2015;205:W374-W381.

31. Collarino A, de Koster EJ, Valdes Olmos RA, de Geus-Oei LF, Pereira Arias-Bouda LM. Is technetium-99m sestamibi imaging able to predict pathologic nonresponse to neoadjuvant chemotherapy in breast cancer? A meta-analysis evaluating current use and shortcomings. Clin Breast Cancer. 2018;18:9-18.

32. Kim S, Plemmons J, Hoang K, et al. Breast-specific gamma imaging versus MRI: comparing the diagnostic performance in assessing treatment response after neoadjuvant chemotherapy in patients with breast cancer. AJR. 2019;212:696-705.

33. Menes TS, Golan O, Vainer G, et al. Assessment of residual disease with molecular breast imaging in patients undergoing neoadjuvant therapy: association with molecular subtypes. Clin Breast Cancer. 2016;16:389-395.

34. Hruska CB, Conners AL, Jones KN, et al. Diagnostic workup and costs of a single supplemental molecular breast imaging screen of mammographically dense breasts. AJR. 2015;204:1345-1353.

35. Adrada BE, Moseley T, Kappadath SC, Whitman GJ, Rauch GM. Molecular breast imaging-guided percutaneous biopsy of breast lesions: a new frontier on breast intervention. J Breast Imaging. 2020;2:484 491.

36. Brem RF, Mehta AK, Rapelyea JA, Akin EA, Bazoberry AM, Velasco CD. Gamma imaging-guided minimally invasive breast biopsy: initial clinical experience. AJR. 2018;210:695-699.

37. Kim G, Bahl M. Assessing risk of breast cancer: a review of risk prediction models. J Breast Imaging. 2021;3:144-155.

38. O'Connor JP, Aboagye EO, Adams JE, et al. Imaging biomarker roadmap for cancer studies. Nat Rev Clin Oncol. 2017;14:169-186.
39. Niell BL, Freer PE, Weinfurtner RJ, Arleo EK, Drukteinis JS. Screening for breast cancer. Radiol Clin North Am. 2017;55:1145-1162.

40. Thompson CM, Mallawaarachchi I, Dwivedi DK, et al. The association of background parenchymal enhancement at breast MRI with breast cancer: a systematic review and meta-analysis. Radiology. 2019;292:552-561.

41. Hruska CB, Scott CG, Conners AL, et al. Background parenchymal uptake on molecular breast imaging as a breast cancer risk factor: a case-control study. Breast Cancer Res. 2016;18:42.

42. Hruska CB, Geske JR, Conners AL, et al. Background parenchymal uptake on molecular breast imaging and breast cancer risk: a cohort study. AJR. 2021;216:1193-1204.

43. Hruska CB, Geske JR, Swanson TN, et al. Quantitative background parenchymal uptake on molecular breast imaging and breast cancer risk: a case-control study. Breast Cancer Res. 2018;20:46.

44. Conners AL, Hruska CB, Tortorelli CL, et al. Lexicon for standardized interpretation of gamma camera molecular breast imaging: observer agreement and diagnostic accuracy. Eur J Nucl Med Mol Imaging. 2012;39:971-982.

45. D'Orsi CJSE, Mendelson EB, Morris EA, et al. ACR BI-RADS® Atlas, Breast Imaging Reporting and Data System. American College of Radiology. 2013;447-458.

46. Hruska CB, Hunt KN, Conners AL, et al. Impact of short-term low-dose tamoxifen on molecular breast imaging background parenchymal uptake: a pilot study. Breast Cancer Res. 2019;21:38.

47. Visvanathan K, Fabian CJ, Bantug E, et al. Use of endocrine therapy for breast cancer risk reduction: ASCO clinical practice guideline update. $J$ Clin Oncol. 2019;37:3152-3165.

48. O'Connor MK, Morrow MMB, Hunt KN, et al. Comparison of Tc-99m maraciclatide and Tc-99m sestamibi molecular breast imaging in patients with suspected breast cancer. EJNMMI Res. 2017;7:5.

49. Bach-Gansmo T, Bogsrud TV, Skretting A. Integrin scintimammography using a dedicated breast imaging, solid-state gamma-camera and ${ }^{99 \mathrm{~m}} \mathrm{Tc}-$ labelled NC100692. Clin Physiol Funct Imaging. 2008;28:235-239. 